\title{
An Operational Investigation of the CPS Hierarchy
}

\author{
Olivier Danvy ${ }^{1}$ and Zhe Yang ${ }^{2}$ * \\ $1 \mathrm{BRICS}$ *** \\ Department of Computer Science, University of Aarhus \\ Building 540, Ny Munkegade, DK-8000 Aarhus C, Denmark \\ danvy@brics.dk \\ 2 Department of Computer Science, New York University \\ 251 Mercer Street, New York, NY 10012, USA \\ zheyang@cs.nyu .edu
}

\begin{abstract}
We explore the hierarchy of control induced by successive transformations into continuation-passing style (CPS) in the presence of "control delimiters" and "composable continuations". Specifically, we investigate the structural operational semantics associated with the CPS hierarchy.

To this end, we characterize an operational notion of continuation semantics. We relate it to the traditional CPS transformation and we use it to account for the control operator shift and the control delimiter reset operationally. We then transcribe the resulting continuation semantics in ML, thus obtaining a native and modular implementation of the entire hierarchy. We illustrate it with several examples, the most significant of which is layered monads.
\end{abstract}

\section{Introduction}

\section{$1.1 \quad$ Background}

Continuation-passing style (CPS) programs are usually obtained by CPS transformation. The CPS hierarchy is obtained by iterating the CPS transformation, which yields programs whose types obey the following pattern:

$$
\begin{aligned}
\text { Fun } & =\text { Val }_{0} \rightarrow \text { Cont }_{1} \rightarrow \text { Cont }_{2} \rightarrow \ldots \rightarrow \text { Cont }_{n} \rightarrow \text { Ans }_{n} \\
\text { Cont }_{1} & =\text { Val }_{1} \rightarrow \text { Cont }_{2} \rightarrow \ldots \rightarrow \text { Cont }_{n} \rightarrow \text { Ans }_{n} \\
& \ldots \\
\text { Cont }_{n} & =\text { Val }_{n} \rightarrow \text { Ans }_{n}
\end{aligned}
$$

In the CPS hierarchy, programs exhibit the familiar pattern of success/failure continuations which is pervasive in functional specifications of backtracking.

* Partially supported by National Science Foundation grant CCR-9616993 and by BRICS.

** Basic Research in Computer Science (http://www.brics.dk), Centre of the Danish National Research Foundation. 
Enriching CPS with identity and composition makes it possible to simulate Prolog-style backtracking and also to accumulate results. To simulate Prologstyle backtracking, we successively apply the current continuation to all possible choices - failing if there are none. To accumulate results, we use the current continuation to compute the result of the "remaining computation" and stash it away in an accumulator. To combine these two control mechanisms, e.g., to accumulate all the possible results of a non-deterministic computation in a list, we exploit the natural hierarchy between these two processes: the generation should take place in a context where its successive results are accumulated. We therefore CPS-transform the generation process (thus making its continuation continuation-passing) and we supply the accumulation process as its initial continuation 5 .

The CPS hierarchy thus offers a fitting platform to express hierarchical backtracking - at the price indicated by the types above: a quadratic inflation of continuations.

The last level of CPS can be avoided by using the identity continuation and the ability to compose continuations. This is often deemed enough when $n=1$ or $n=2$ in the type equations above. For higher values of $n$, this quadratic cost can be alleviated with a linguistic device: two new syntactic forms in direct style, whose CPS transformation yields the desired effect of initializing a continuation with identity and of composing continuations. Initializing a continuation with identity is achieved with the control delimiter reset. Composing continuations is enabled by the control operator shift which captures the current (delimited) continuation and makes it ready to be composed with a subsequent continuation 56 . For comparison, the control operator callcc captures the current (unlimited) continuation and makes it ready to replace a subsequent continuation 1723 .

The challenge now is how to implement the CPS hierarchy more directly than by repeated CPS transformations and more efficiently than with a definitional interpreter 5. Filinski showed how to implement the first level natively, using callcc and one reference cell 12 . In this article, we show how to implement the entire hierarchy natively, using callcc and one reference cell per level.

\subsection{Related work}

The CPS hierarchy was identified and advocated by Danvy and Filinski 5, who also introduced the corresponding hierarchy of control operators shift $_{n}$ and $\operatorname{reset}_{n}$ (one per surrounding continuation). At the same time, but independently of CPS, Felleisen invented control delimiters $\boldsymbol{x}$, initiating a whole area of work on composable continuations and hierarchies of control 9 II If if $1921 \% 27$, 313.3 .34 . Control delimiters, for example, were instrumental to obtain a fullabstraction result 3.5 .

All researchers in this new area followed Felleisen and defined their new control constructs operationally. They reported a variety of control operators, each of these displaying inventiveness in its modus operandi, its description, and its implementation. 
In contrast, shift and reset are defined by translation into CPS. They have also proven particularly fruitful: because (we believe) control delimiters and composable continuations arise naturally in the CPS hierarchy, a number of applications of shift and reset were reported through the 90's 41214242537 , up to and including the R5RS 23. There were only two further studies, however, of the CPS hierarchy and its guidelines: Murthy's, formalizing its type system 28, and Filinski's, establishing its equivalence with computational monads 1315 .

\subsection{This work}

The growing number of applications of shift and reset leads one to want to combine them. For example, suppose that we want to specialize programs that use shift and reset, using type-directed partial evaluation 4. The problem is that type-directed partial evaluation also uses shift and reset, and we would like these two uses not to interfere with each other. This kind of applications require the CPS hierarchy to layer different uses of shift and reset at different levels.

It is difficult to implement the CPS hierarchy natively, since the semantics of built-in constructs cannot be altered. We thus take a novel approach using operational semantics: we characterize an operational 'continuation semantics' and following Section 1.] (1) we enrich it with identity and composition of continuation as provided by shift and reset, and (2) we transform the result in a new continuation semantics. The new semantics extends the old one with a new pair of shift and reset; moreover, it can be natively implemented in the old semantics, since all the rules in the new semantics except those of the newly added operators are those of the old semantics, with the addition of one unchanged component. Iterating this process yields a family of semantics - the CPS hierarchy - and its native and modular implementation in ML, à la Filinski I2 I.3 I5. This general approach provides a native implementation of the new language constructs.

En passant, to make sure that we account for shift and reset as originally defined (i.e., by CPS transformation), we relate our operational notion of continuation semantics with the traditional CPS transformation.

\subsection{Applications}

A toy example: The two following computations declare an outer context $1+$ [ ]. They also declare a delimited context $[50+[]]$, which is abstracted as a function denoted by $\mathrm{k}$. This function is successively applied to 0 , yielding 50, and to 10, yielding 60. These two results are added, yielding 110 which is then plugged in the outer context. In both cases, the overall result is 111.

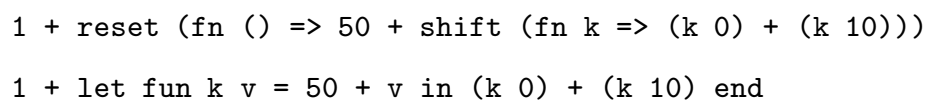

In the first computation, the context is delimited by reset and the delimited context is abstracted into a function with shift. The second computation is the continuation-passing counterpart of the first one. 
More substantial examples: The CPS hierarchy also makes it possible to express computations like min-max processes or quantifier alternation. For example, the existential quantifier $\exists v \cdot p(v)$ for a condition $p$ over non-deterministically generated values $v$ can be implemented as

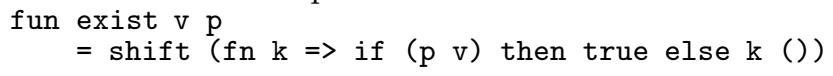

where the return value of reset corresponding to the collection is set to false. Similarly, we can implement the universal quantifier with a function forall. Using these two functions at different levels, we can write formulae of arbitrary quantifier alternation.

\section{Operational Semantics of the CPS Hierarchy}

This section presents a family of operational semantics that can be directly transcribed into an implementation.

Starting with an operational semantics $S$ for ML (Section 2.I , we characterize an operational notion of continuation semantics (Section $2 \cdot 2$. We then relate continuation semantics and syntactic CPS transformation, which is a result in itself (Sections 2.3 and 2.4. Based on the CPS transformation, we provide a semantic account of shift and reset (Section 25 .

The semantics $L$, which is $S$ extended with shift and reset, is no longer a continuation semantics. We induce two continuation semantics $H$ and $I$, and prove that they both simulate the semantics $L$ (Sections $\mathbf{2 . 6}$ and $\mathbf{2 . 4}$. Moreover, $I$ is directly implementable in $S$, in that the $S$-rules embed into the $I$-rules with the addition of one unchanged component. This component can be implemented by a reference cell. As for the remaining $I$-rules, they correspond to new control operators which can be implemented as functions.

The resulting semantics $I$ is a continuation semantics, and thus, generalizing, we can iterate the whole transformation (Section 2.8. The resulting family of operational semantics formalizes the CPS hierarchy and is directly implementable in the initial operational semantics of ML (Section 3 .

\subsection{Starting semantics $S$}

We use Harper, Duba, and MacQueen's "continuation-based operational semantics" for ML 17 as our starting semantics $S^{1}$ Its syntactic categories are defined by the following grammar.

$$
\begin{array}{rlrl}
e \in \operatorname{Exp} & ::=x|\ell| \lambda x . e \mid e_{0} e_{1} & & \text {-expressions } \\
v \in \text { Val }::=\ell \mid \lambda x . e & & \text {-values } \\
k \in \text { Cont } & :=\square|k e| v k & & \text { - continuations }
\end{array}
$$

Its inference rules specify a judgment of the form " $k \vdash e \Rightarrow v$ " which reads "under the continuation $k$, evaluating the expression $e$ yields the answer $v$ " (Table II).

${ }^{1}$ For brevity, both WRONG and LET rules in the original semantics are omitted here. The WRONG rule specifies the error case and serves in the formulation of type soundness. The LET rule is only there for ML's let polymorphism. 


$$
\begin{aligned}
& (\mathrm{VAL0}) \frac{\square \vdash v \Rightarrow v}{\square \vdash v\left[v_{1}\right] \Rightarrow v} \quad(k \neq \square) \\
& (\mathrm{FN}) \frac{k\left[\square e_{1}\right] \vdash e_{0} \Rightarrow v}{k \vdash e_{0} e_{1} \Rightarrow v}\left(e_{0} \text { not a value }\right) \quad(\mathrm{ARG}) \frac{k\left[v_{0} \square\right] \vdash e_{1} \Rightarrow v}{k \vdash v_{0} e_{1} \Rightarrow v}\left(e_{1} \text { not a value }\right) \\
& (\mathrm{BETA}) \frac{k \vdash\left[v_{1} / x\right] e \Rightarrow v}{k \vdash(\lambda x . e) v_{1} \Rightarrow v}
\end{aligned}
$$

Table 1. An operational continuation semantics

A continuation $k$ can be thought of as an expression with precisely one "hole" $\square$ in it. We write $k[e]$ and $k\left[k^{\prime}\right]$ to denote the expression and continuation obtained by filling the hole in $k$ with an expression $e$ and a continuation $k^{\prime}$, respectively, where $k\left[k^{\prime}\right]$ is the "composition" of $k$ with $k^{\prime}$.

We are mainly interested in the dynamic semantics of shift and reset, and thus we do not present typing rules and the related soundness proof, which can be adapted from the work of Harper, Duba, and MacQueen 17 and of Gunter, Rémy, and Riecke 16 . We rely on the static type system of our implementation language, ML, for the type soundness (Section $\mathbf{3}$.

\subsection{An operational notion of continuation semantics}

Operational semantics give rise to derivation trees. We define a branchless semantics as an operational semantics whose rules have one premise at most. Such a semantics gives rise to branchless derivation trees, i.e., lists. Note that a branchless semantics directly corresponds to a reduction semantics, where reduction proceeds from the conclusion of a rule to the premise, or to the final result if the rule has no premise. Staying in the world of branchless evaluation semantics makes it easy to refer to a complete computation (as a judgment) as well as a single reduction (as a rule instance).

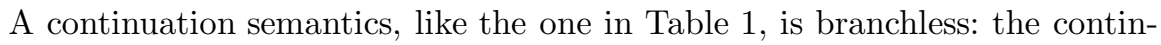
uation component in its judgments keeps track of the remaining branches in a corresponding direct-style derivation tree; it can be regarded as the stack used to traverse this derivation tree.

\subsection{CPS transformation}

Previous studies of the CPS hierarchy build on the CPS transformation. To justify our study of control operators with the continuation semantics of Table II we adapt the call-by-value CPS transformation to its expressions, values, and continuations.

$$
\begin{aligned}
& \llbracket x \rrbracket_{E x p}=\lambda \kappa . \kappa x \\
& \llbracket v \|_{E x p}=\lambda \kappa . \kappa \llbracket v \rrbracket V a l
\end{aligned}
$$

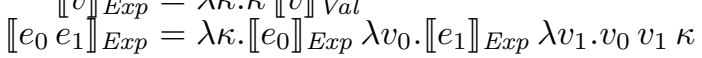

$$
\begin{aligned}
& \begin{aligned}
\llbracket \ell \rrbracket_{V a l} & =\ell \\
\llbracket \lambda x . e \rrbracket \text { Val } & =\lambda x . \llbracket e \rrbracket_{E x p}
\end{aligned}
\end{aligned}
$$




$$
\begin{aligned}
\llbracket \square \rrbracket & \prod_{\text {Cont }}=\lambda v \cdot \lambda \kappa \cdot \kappa v \\
\llbracket k e_{1} \rrbracket_{\text {Cont }} & =\lambda v \cdot \lambda \kappa \cdot \llbracket k \rrbracket_{\text {Cont }} v \lambda v_{0} \cdot \llbracket e_{1} \rrbracket_{\text {Exp }} \lambda v_{1} \cdot v_{0} v_{1} \kappa \\
\llbracket v_{0} k \rrbracket_{\text {Cont }} & =\lambda v \cdot \lambda \kappa \cdot \llbracket k \rrbracket_{\text {Cont }} v \lambda v_{1} \cdot \llbracket v_{0} \rrbracket_{\text {Val }} v_{1} \kappa
\end{aligned}
$$

The rationale for $\llbracket \cdot \rrbracket_{\text {Cont }}$ is that the "hole" $\square$ in the continuation is an abstraction over a value, as captured in the following lemma, where " $=\beta \eta$ " denotes $\beta \eta$-convertibility.

Lemma 1. For all $k \in$ Cont and $v \in$ Val, $\llbracket k[v] \rrbracket_{E x p}={ }_{\beta \eta} \llbracket k \rrbracket_{\text {Cont }} \llbracket v \rrbracket_{\text {Val }}$.

Proof. By structural induction on $k$.

\subsection{Soundness and completeness of the operational semantics}

The following theorem connects the continuation semantics of Section 2 ll and the CPS transformation of Section 2.3

Theorem 1. For all $k \in$ Cont, $e \in \operatorname{Exp}$, and $v \in V a l$, if $k \vdash e \Rightarrow v$ then

$$
\llbracket e \rrbracket_{E x p} \lambda w \cdot \llbracket k \rrbracket_{C o n t} w \lambda a \cdot a=\beta \eta \llbracket v \rrbracket_{\text {Val }} .
$$

Proof. By rule induction on $k \vdash e \Rightarrow v$.

Corollary 1. For all $e \in \operatorname{Exp}$ and $v \in V a l$, if $\square \vdash e \Rightarrow v$ then $\llbracket \rrbracket_{\text {Exp }} \lambda w . w$ $=\beta \eta \llbracket v \rrbracket_{V a l}$.

Because the term $\llbracket e \rrbracket_{\operatorname{Exp}} \lambda w . w$ is convertible to a value $\llbracket v \rrbracket_{V a l}$, it must reduce to a value that is equal to $\llbracket v \rrbracket_{V a l}$ modulo $\beta \eta$-conversion under normal-order evaluation (by the Normalization theorem), and thus also under applicative-order evaluation (by Plotkin's Indifference theorem 29). The operational semantics is thus sound with respect to the call-by-value semantics defined by the CPS transformation.

Proving completeness requires a close correspondence between the rules and the translated terms, and as often, "administrative redexes" in the CPS transformation get in the way. To prove completeness (i.e., that the evaluation of the CPS form of a term $e$ with an identity continuation leads to a value $v$, then $\square \vdash e \Rightarrow v$ ), we successfully adopted Danvy and Filinski's one-pass CPS transformation 6 .

These soundness and completeness results are not surprising. One can also obtain them by proving the equivalence of the continuation semantics and a direct semantics, and then by using Plotkin's Simulation theorem 29. A more immediate connection between continuation semantics and CPS transformation, however, provides the basic framework for adding control operators.

\subsection{An operational account of shift and reset}

A control operator "reifies" a continuation $k$ into a function $f_{k}$. In terms of the CPS transformation, such a function appears as a $\lambda$-term:

$$
\Lambda_{k}=\lambda w \cdot \llbracket k \rrbracket_{\text {Cont }} w \lambda a . a .
$$


Correspondingly, in the continuation semantics, we need to find out how to invoke such a function $f_{k}$ to be able to use a reified continuation.

Let us fix a continuation $k$. For any given value $v$, the corresponding $v^{\prime}$ such that $k \vdash v \Rightarrow v^{\prime}$ is unique, if it exists. This suggests us to define a function $f_{k}$ for every continuation $k$ as follows.

$$
f_{k} v=v^{\prime} \Longleftrightarrow k \vdash v \Rightarrow v^{\prime}
$$

And this is justified by the CPS transformation: as a corollary of Theorem II when $e=v$, the term $\Lambda_{k}\left(\llbracket v \rrbracket_{\text {Val }}\right)$ is $\beta \eta$-convertible to

$$
\left(\lambda w \cdot \llbracket k \rrbracket_{C o n t} w \lambda a \cdot a\right) \llbracket v \rrbracket_{V a l}=\beta \eta \llbracket v \rrbracket_{E x p} \lambda w \cdot \llbracket k \rrbracket_{C o n t} w \lambda a \cdot a={ }_{\beta \eta} \llbracket v^{\prime} \rrbracket_{V a l} .
$$

Now we are ready to introduce the control operators shift and reset by adding the following expression forms and the corresponding rules.

$$
e::=\ldots \mid \text { reset } e \mid \text { shift } c . e \mid \operatorname{pushcc}(e, k)
$$

Shift and reset are defined by their CPS transformation 56 :

$$
\begin{aligned}
\llbracket \text { shift } c . e \rrbracket_{E x p}= & \lambda \kappa \cdot\left(\lambda c \cdot \llbracket e \rrbracket_{E x p} \lambda a \cdot a\right) \lambda w \cdot \lambda \kappa^{\prime} \cdot \kappa^{\prime}(\kappa w) \\
& -\operatorname{composition} \text { of continuations } \\
\llbracket \text { reset } e \rrbracket_{E x p}= & \lambda \kappa \cdot \kappa\left(\llbracket e \rrbracket_{E x p} \lambda a \cdot a\right) \\
& - \text { identity continuation }
\end{aligned}
$$

In both shift c.e and reset $e$, the type of $e$ should be the same - though not necessarily the same as the final result type. Thus any shift-expression must be delimited by a corresponding reset or a shift - a hidden restriction that cannot be easily expressed in this translation. We address it in Section 2.6

The translation of shift and reset are not in CPS because they compose continuations. This programming pattern is abstracted by the meta-control operator pushcc:

$$
\llbracket \operatorname{pushcc}(e, k) \rrbracket_{E x p}=\lambda \kappa . \kappa\left(\llbracket e \rrbracket_{E x p} \lambda w . \llbracket k \rrbracket_{C o n t} w \lambda a . a\right)
$$

Pushcc is a meta-control operator because its expression form contains continuations. It therefore can only be used to define other control operators.

Correspondingly, in the operational semantics, we can express the composition of functions $f_{k}$ and $f_{k}^{\prime}$ related to continuations $k$ and $k^{\prime}$ by adding the rule pushcc (Table 2 . As for shift and reset, they are defined by the rules shift and reset in term of pushcc.

Let us resume the inductive proof of Theorem II for the three new rules. We only reproduce the most interesting one here, i.e., pushcc.

\section{Proof. (excerpt)}

The induction hypotheses for the rule pushcc (Table 2 read:

$$
\begin{aligned}
& \llbracket e \rrbracket_{\operatorname{Exp}} \lambda w \cdot \llbracket k^{\prime} \rrbracket_{C o n t} w \lambda a \cdot a={ }_{\beta \eta} \llbracket v^{\prime} \rrbracket_{\text {Val }} \\
& \llbracket v^{\prime} \rrbracket_{\text {Exp }} \lambda w \cdot \llbracket k \rrbracket_{\text {Cont }} w \lambda a \cdot a={ }_{\beta \eta} \llbracket k \rrbracket_{\text {Cont }} \llbracket v^{\prime} \rrbracket_{\text {Val }} \lambda a \cdot a={ }_{\beta \eta} \llbracket v \rrbracket_{\text {Val }}
\end{aligned}
$$




$$
\begin{gathered}
(\text { shift }) \frac{\square \vdash(\lambda c . e) \lambda w \cdot \operatorname{pushcc}(w, k) \Rightarrow v}{k \vdash \operatorname{shift} c . e \Rightarrow v} \quad \text { (reset) } \frac{k \vdash \operatorname{pushcc}(e, \square) \Rightarrow v}{k \vdash \operatorname{reset} e \Rightarrow v} \\
\text { (pushcc) } \frac{k^{\prime} \vdash e \Rightarrow v^{\prime} \quad k \vdash v^{\prime} \Rightarrow v}{k \vdash \operatorname{pushcc}\left(e, k^{\prime}\right) \Rightarrow v}
\end{gathered}
$$

Table 2. Definitional rules for shift and reset

Now, we have $\llbracket \operatorname{pushcc}\left(e, k^{\prime}\right) \rrbracket_{\text {Exp }} \lambda w \cdot \llbracket k \rrbracket_{\text {Cont }} w \lambda a . a$

$$
\begin{aligned}
& =\beta \eta\left(\lambda \kappa \cdot \kappa\left(\llbracket e \rrbracket_{E_{x p}} \lambda w \cdot \llbracket k^{\prime} \rrbracket_{\text {Cont }} w \lambda a \cdot a\right)\right) \lambda w \cdot \llbracket k \rrbracket_{\text {Cont }} w \lambda a . a \\
& =\beta \eta \llbracket k \rrbracket_{\text {Cont }} \llbracket v^{\prime} \rrbracket_{\text {Val }} \lambda a \cdot a, \text { by (i1) } \\
& =\beta \eta \llbracket v \rrbracket_{\text {Val }}, \text { by (i2) }
\end{aligned}
$$

Theorem II and consequently Corollary II still hold for the operational semantics and the CPS transformation extended with the control operators. Therefore, the operational semantics gives the same definition for the control operators as those given by the CPS transformation.

\subsection{A continuation semantics for shift and reset}

With the addition of pushcc, the semantics is no longer branchless. To obtain an equivalent branchless semantics, we need to use the idea of the CPS transformation, i.e., flattening derivation trees by remembering branching computations (continuations $k$ in pushcc) in a stack.

We thus induce another semantics $H$ from the extended semantics, referred to as $L$. For clarity, we subscript rules and domains by the semantics they belong to. A judgment in $H$ is of the form $k \vdash_{H} \quad e \Rightarrow v$, where $e \in \operatorname{Exp}_{L}$ (which can be one of the form introduced by the control operators), but $k$ forms a new domain of "global" continuations: $k \in \operatorname{Cont}_{H}=\operatorname{Cont}_{L} \times\left(\right.$ Cont $_{L}$ list $)=$ $\operatorname{Cont}_{L}^{+}$. The global continuations (of type $\operatorname{Cont}_{H}$ ) are always non-empty lists of continuations, whose head is the current active continuation, and whose tail is a stack of saved continuations.

The $H$-rules are given in Table 3 Most of them are simply the corresponding $L$-rules with the stack $k s$ carried around unchanged. The interesting rules, pushcc $_{\mathrm{H}}$ and $\mathrm{VALO}_{\mathrm{H}}^{\mathrm{CONS}}$, function as the branching rule pushcc $\mathrm{L}_{\mathrm{L}}$.

The semantics $H$ is branchless. We would like to show that it correctly accounts for $L$, i.e., $\forall k, e, v$. $\left(k \vdash_{L} e \Rightarrow v\right) \Longleftrightarrow\left(k::\right.$ nil $\left.\vdash_{H} e \Rightarrow v\right)$.

Theorem 2. For all $k \in \operatorname{Cont}_{L}, e \in \operatorname{Exp}_{L}$ and $v \in V_{a l}$, if $k \vdash_{L} e \Rightarrow v$, then $\forall k s \in \operatorname{Cont}_{L}^{*}, v^{\prime} \in \operatorname{Val}_{L} \cdot\left(\square:: k s \vdash_{H} v \Rightarrow v^{\prime}\right) \Longrightarrow\left(k:: k s \vdash_{H} e \Rightarrow v^{\prime}\right)$.

Proof. By rule induction on $k \vdash_{L} e \Rightarrow v$.

For $k s=$ nil, using rule $\mathrm{VALO}_{\mathrm{H}}^{\mathrm{NIL}}$, we obtain the following corollary.

Corollary 2. For all $k \in \operatorname{Cont}_{L}, e \in \operatorname{Exp}_{L}$ and $v \in \operatorname{Val}_{L}$, if $k \vdash_{L} e \Rightarrow v$ then $k::$ nil $\vdash_{H} e \Rightarrow v$. 


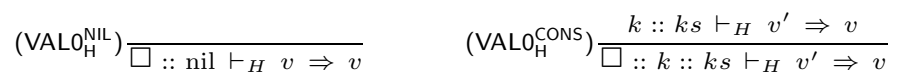

$$
\begin{aligned}
& \left(\mathrm{VAL1}_{H}\right) \frac{\square:: k s \vdash_{H} k\left[v_{1}\right] \Rightarrow v}{k:: k s \vdash_{H} v_{1} \Rightarrow v}(k \neq \square) \quad(\mathrm{FN}) \frac{k\left[\square e_{1}\right]:: k s \vdash_{H} e_{0} \Rightarrow v}{k:: k s \vdash_{H} e_{0} e_{1} \Rightarrow v}\left(e_{0} \text { not a value }\right) \\
& \left(\mathrm{ARG}_{H}\right) \frac{k\left[v_{0} \square\right]:: k s \vdash_{H} e_{1} \Rightarrow v}{k:: k s \vdash_{H} v_{0} e_{1} \Rightarrow v}\left(e_{1} \text { not a value }\right) \quad\left(\mathrm{BETA}_{H}\right) \frac{k:: k s \vdash_{H}\left[v_{1} / x\right] e \Rightarrow v}{k:: k s \vdash_{H}(\lambda x . e) v_{1} \Rightarrow v} \\
& \left(\text { shift }_{H}\right) \frac{\square:: k s \vdash_{H}(\lambda c . e) \lambda w \cdot \operatorname{pushcc}(w, k) \Rightarrow v}{k:: k s \vdash_{H} \operatorname{shift} c . e \Rightarrow v}\left(\operatorname{reset}_{H}\right) \frac{k:: k s \vdash_{H} \operatorname{pushcc}(e, \square) \Rightarrow v}{k:: k s \vdash_{H} \operatorname{reset} e \Rightarrow v} \\
& \text { (pushcc } \left.{ }_{H}\right) \frac{k:: k^{\prime}:: k s \vdash_{H} e \Rightarrow v}{k^{\prime}:: k s \vdash_{H} \operatorname{pushcc}(e, k) \Rightarrow v}
\end{aligned}
$$

Table 3. An operational continuation semantics for shift and reset

For the inverse direction, we need to refer to the derivation more explicitly: we use $\preceq$ to denote the sub-derivation relation, and we write $D: J$ if $D$ is a derivation ending with the judgment $J$.

Theorem 3. For all $k s \in \operatorname{Cont}_{L}^{*}, k \in \operatorname{Cont}_{L}, e \in \operatorname{Exp}_{L}, v^{\prime} \in \operatorname{Val}_{L}$, if D : $k:: k s \vdash_{H} e \Rightarrow v^{\prime}$, then

$$
\exists v \in \operatorname{Val}_{L}, D^{\prime} .\left(k \vdash_{L} e \Rightarrow v\right) \wedge\left(D^{\prime} \preceq D\right) \wedge D^{\prime}:\left(\square:: k s \vdash_{H} v \Rightarrow v^{\prime}\right) .
$$

Proof. By strong induction on the derivation $D$.

For $k s=$ nil in Theorem 3 we notice that the only possible derivation for $D^{\prime}$ is one-step, using rule $\mathrm{VALO}_{\mathrm{H}}^{\mathrm{NIL}}$, so the witness $v$ is $v^{\prime}$, and the following corollary holds.

Corollary 3. For all $k \in$ Cont $_{L}, e \in \operatorname{Exp}_{L}$ and $v \in \operatorname{Val}_{L}$, if $k::$ nil $\vdash_{H} e \Rightarrow v$ then $k \vdash_{L} e \Rightarrow v$.

Together, Corollary $₫$ and Corollary 3 show that the branchless semantics $H$ simulates the semantics $L$. $H$ can be implemented by a definitional interpreter as before 5 . Our goal, however, is to implement the control operators natively as functions (using first-class continuations and cells, like Filinski 12 ). The semantics of the built-in constructs cannot be altered in such a setting, thereby preventing us to implement the crucial rule VALO ${ }_{\mathrm{H}}^{\mathrm{CONS}}$, which is enacted when the active continuation (of type Cont $_{L}$ ) is already identity. Such behavior should be put into the continuation: instead of initializing it with an identity continuation $\square$, we should initialize it with an operation to resume the top continuation from the stack. The corresponding transformation of $L$ is described in Section 2.7

Now we can come back to the typing problem of shift. The requirement that all shift-expressions must be enclosed by a corresponding reset or shift can be easily manifested in the semantics $H$ by adding a side condition to the rule 


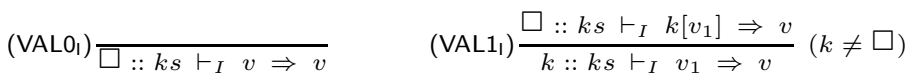

$$
\begin{aligned}
& \left(\mathrm{FN}_{\mathrm{I}}\right) \frac{k\left[\square e_{1}\right]:: k s \vdash_{I} e_{0} \Rightarrow v}{k:: k s \vdash_{I} e_{0} e_{1} \Rightarrow v}\left(e_{0} \text { not a value }\right) \\
& (\mathrm{ARG}) \frac{k\left[v_{0} \square\right]:: k s \vdash_{I} e_{1} \Rightarrow v}{k:: k s \vdash_{I} v_{0} e_{1} \Rightarrow v}\left(e_{1} \text { not a value }\right) \quad\left(\mathrm{BETA}_{\mathrm{I}}\right) \frac{k:: k s \vdash_{I}\left[v_{1} / x\right] e \Rightarrow v}{k:: k s \vdash_{I}(\lambda x . e) v_{1} \Rightarrow v} \\
& \left(\text { shift }_{1}\right) \frac{i d_{L}^{\text {pop }}:: k s \vdash_{I}(\lambda c . e) \lambda w \cdot \operatorname{pushcc}(w, k) \Rightarrow v}{k:: k s \vdash_{I} \text { shift } c . e \Rightarrow v}(k s \neq \text { nil }) \\
& \left(\text { reset }_{1}\right) \frac{k:: k s \vdash_{I} \operatorname{pushcc}\left(e, i d_{L}^{p o p}\right) \Rightarrow v}{k:: k s \vdash_{I} \operatorname{reset} e \Rightarrow v} \quad\left(\text { pushcc }_{1}\right) \frac{k:: k^{\prime}:: k s \vdash_{I} e \Rightarrow v}{k^{\prime}:: k s \vdash_{I} \operatorname{pushcc}(e, k) \Rightarrow v} \\
& \left(\operatorname{popcc}_{1}\right) \frac{k s \vdash_{I} v \Rightarrow v^{\prime}}{k^{\prime}:: k s \vdash_{I} \operatorname{popcc}(v) \Rightarrow v^{\prime}}(k s \neq \text { nil })
\end{aligned}
$$

Table 4. An implementable semantics for shift and reset

shift $_{\mathrm{H}}$ that the stack should not be empty. (This makes the definition of shift partial; when the stack is empty, we obtain a run-time error.)

$$
\left(\operatorname{shift}_{\mathrm{H}}\right) \frac{\square:: k s \vdash_{H}\left(\lambda k^{\prime} . e\right) \lambda w \cdot \operatorname{pushcc}(w, k) \Rightarrow v}{k:: k s \vdash_{H} \text { shift } k^{\prime} . e \Rightarrow v}(k s \neq \operatorname{nil})
$$

\subsection{An implementable continuation semantics for shift and reset}

The implementable semantics $I$ is also induced from the semantics $L$ with Cont $_{I}$ $=$ Cont $_{H}=$ Cont $_{L}^{+}$. We introduce a new operator popcc:

$$
e::=\ldots \mid \operatorname{popcc}(e)
$$

Intuitively, this operator pops the continuation stack and sends its operand to the popped continuation. Now, the initial continuation can be defined as $i d_{L}^{\text {pop }} \stackrel{\text { def }}{=}$ popcc $(\square)$, which replaces $\square$ in rules shift ${ }_{\mathrm{H}}$ and reset ${ }_{\mathrm{H}}$, thus eliminating the need for the rule VALO $\mathrm{H}_{\mathrm{H}}^{\mathrm{CONS}}$.

In Table 4 the $I$-rules are the same as the $H$-rules except for VALO (replacing $\mathrm{VALO}_{\mathrm{H}}^{\mathrm{NIL}}$ and $\mathrm{VALO}_{\mathrm{H}}^{\mathrm{CONS}}$ ), shift $_{\mathrm{l}}$, reset $_{\text {, }}$, and popcc $_{\text {I }}$.

Semantics $I$ simulates semantics $L$, as shown by the following theorem.

Theorem 4. For all $e \in \operatorname{Exp}_{L}$ and $v \in \operatorname{Val}_{L},\left(\square::\right.$ nil $\left.\vdash_{I} e \Rightarrow v\right) \Longleftrightarrow$ $\left(\square::\right.$ nil $\vdash_{H} e \Rightarrow v$ ).

Proof. By two straightforward inductions.

This new semantics $I$ has two properties:

(1) It is branchless. More specifically, for any intermediate judgment $k \vdash_{I}$ $e \Rightarrow v$ in a proof tree, the rest of the computation after $e$ is evaluated is totally captured in the global continuation $k$. We can thus iterate the above process to add control operators at subsequent levels. 
(2) It can be directly implemented in the starting semantics $S$ using references and first-class continuations in the following way $t$ the head of the global continuation $k$ is the current continuation, while the tail is stored in a reference cell. All the $S$-rules automatically 'extend' to the corresponding $I$-rules, without touching the reference cell; the new rules defining the control operators can then be directly implemented by encoding the four constants shift, reset, pushcc and popcc as functions, using callcc to capture the current continuation and throw to restore it.

\subsection{An inductive construction of the CPS hierarchy}

The semantic transformation (from Section 2.5 to Section $\mathbf{2}$. can be generalized and iterated: at each step, we transform an input semantics $S_{i}$ into an output semantics $S_{i+1}$ that preserves certain inductive conditions (such as "branchlessness"). The operational continuation semantics displayed in Table II satisfies these inductive conditions, and we used it as the starting semantics $S_{1}$.

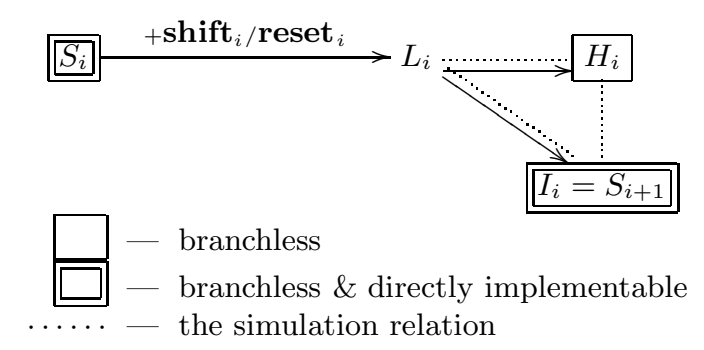

Fig. 1. A single transformation (from level $i$ to level $i+1$ )

Figure II summarizes the development of a single transformation: we start with the branchless semantics $S_{i}$ of level $i$. Adding shift $i$ and reset $_{i}$ with related inference rules yields the semantics $L_{i}$ which is no longer branchless (see Section 2.5 . Then we replace the global continuation of this level by a stack of such continuations, which forms the global continuation of the next level, and we obtain a semantics $H_{i}$ where we restore the branchlessness property (see Section 2.6. Since $H_{i}$ is not directly implementable in $S_{i}$, we apply another transformation to $L_{i}$ to obtain a semantics $I_{i}$, which is both branchless and directly implementable (see Section 2.7. Semantics $I_{i}$ simulates $H_{i}$, which in turn simulates $L_{i}$. With its newly introduced control operators $\operatorname{shift}_{i}$ and $\operatorname{reset}_{i}, I_{i}$ satisfies the inductive conditions. Therefore, we can use it as the semantics $S_{i+1}$ for the next level of the hierarchy.

${ }^{2}$ We did not put references and callcc in the starting semantics and we only use them for the implementation. In fact, making references available to the user causes no problem, whereas callcc interferes with shift and reset. 
For space reasons, the rest of this section is omitted. But it is available in the extended version of this article 7 .

\section{An Implementation of the CPS Hierarchy}

We implement the CPS hierarchy by transcribing the transformation of Section 28 in Standard ML of New Jersey I, using the structure SMLofNJ. Cont which provides callcc and throw. The implementation uses a signature SHIFT_ RESET to specify the operations provided by a semantics $S$ for the user and for the construction of the next control level, a structure innermost level to model the first level in the hierarchy (which thus provides no control operators), and a functor sr_outer to construct next-level control operators, parameterized by the answer type ans and by the control level inner immediately preceding it. Essentially, the signature SHIFT_RESET corresponds to the inductive conditions for the semantics $S$, and the functor sr_outer corresponds to the transformation from a semantics $S=S_{i}$ to the next-level semantics $I=S_{i+1}$.

The implementations of control operators are thus hidden inside the module system, and they are accessed via the name of the structure that corresponds to their level. Having devised an ordering of the control effects, a user then implements it through the order of functor applications. Hierarchical occurrences of shift and reset are thus no longer referred to by their relative index, which had been criticized in the literature $16 \mathrm{2} / \mathrm{T}$.

We implemented the functor sr_outer by transcribing line-by-line the added semantic rules in semantics $I$ (four new functions, one per operator) and the definition of the constant $i d_{L}^{p o p} \mathbf{3}$ We also use two auxiliary functions: a function replace_gcont, used implicitly in the semantics, captures and replaces the current global continuation, and a function cont2gcont, required by the inductive conditions for semantics $S$, converts a first-class continuation to a global continuation. The code is thus very concise: the pretty-printed program defining innermost_level and sr_outer takes about 40 lines of ML code (Figure $\mathbf{3}$.

We also provide a functor for the usual first level of control operators ( shift $_{1}$ and $\left.\operatorname{reset}_{1}\right)$ :

functor initial_control_level (type ans) : SHIFT_RESET

$=$ sr_outer (type ans = ans structure inner = innermost_level)

Specializing this functor for the first level of the CPS hierarchy yields a result similar to Filinski's implementation of shift and reset 12 . The main difference is that here we use an explicit stack of continuations whereas Filinski uses an implicit one through functional abstraction. (An analogy: one can represent environments in an interpreter as a list or as a function.)

\footnotetext{
${ }^{3}$ We use the function SMLofNJ. Cont.isolate to coerce a non-returning function to a continuation. This function can be defined as follows.
}

fun isolate $f=\operatorname{callcc}(f n x \Rightarrow f(\operatorname{callcc}($ fn $y \Rightarrow$ throw $x y)))$ 
signature SHIFT_RESET

$=$ sig

type answer

val reset : (unit $\rightarrow$ answer) $\rightarrow$ answer

val shift : (('a $\rightarrow$ answer) $\rightarrow$ answer) $\rightarrow$ 'a

type 'a gcont

val replace_gcont : 'a gcont $\rightarrow$ ('b gcont $\rightarrow$ 'a) $\rightarrow$ 'b

(* captures current global continuation (of type 'b gcont), *)

(* and replaces it with the first argument (of type ' $a$ gcont) *)

val cont2gcont : 'a cont $\rightarrow$ 'a gcont

$\left(* \operatorname{Cont}_{S}^{0} \rightarrow\right.$ Cont $\left._{S}^{k} *\right)$ end

structure innermost_level :> SHIFT_RESET

= struct

exception InnermostLevelNoControl

type answer $=$ unit

type 'a gcont $=$ 'a cont

$(*$ level $0 *)$

(* control level $i *)$

(* answer type of level $i^{*}$ )

(* $\left.\operatorname{Cont}_{S}^{i}\left(=\operatorname{Cont}_{L}^{i}\right) *\right)$

fun replace_gcont new_c e_thunk

$=$ callcc (fn old_c $\Rightarrow$ throw new_c (e_thunk old_c))

fun cont2gcont $c=c$

fun reset _ = raise InnermostLevelNoControl

fun shift _ = raise InnermostLevelNoControl

end

functor sr_outer (type ans structure inner: SHIFT_RESET) :> SHIFT_RESET

where type answer $=$ ans $\quad\left({ }^{*}\right.$ from $S=S_{i}$ to $\left.I=S_{i+1} *\right)$

$=$ struct

exception MissingReset

exception Fatal

type answer $=$ ans

type 'a gcont

$=$ (answer inner.gcont) list * 'a inner.gcont

$\left({ }^{*} \operatorname{Cont}_{I}=\operatorname{Cont}_{S}^{+*}\right)$

val stack $=$ ref []$:$ (answer inner.gcont) list ref

$(* \quad k s *)$

fun replace_gcont (new_ks, new_k) e_thunk

$=$ inner.replace_gcont new_k

(* captures and replaces the global continuation, recursively *)

( $\mathrm{fn}$ cur_k $\Rightarrow$ let val cur_gcont $=$ (! stack, cur_k)

in stack := new_ks; (e_thunk cur_gcont) end)

fun cont2gcont action

$=([]$, inner.cont2gcont action $)$

fun popcc $v$

= case !stack of

[] $\Rightarrow$ raise Fatal

| $\mathrm{k}^{\prime}:: \mathrm{ks} \Rightarrow$ (stack := ks; inner.replace_gcont k' (fn _ $\left.\Rightarrow \mathrm{v}\right)$ )

val id_popcc $=$ inner. cont2gcont (isolate popcc)

fun pushcc $k$ e_thunk

$=$ inner.replace_gcont $\mathrm{k}$

(fn $k^{\prime} \Rightarrow$ (stack $:=k^{\prime}::$ !stack; e_thunk ()))

fun reset $e_{-}$thunk

$=$ pushcc id_popcc e_thunk

fun shift k_abstraction

= case ! stack of

[] $\Rightarrow$ raise MissingReset

I _ $\Rightarrow$ inner.replace_gcont id_popcc

(fn ( $\mathrm{k}:$ ' $a$ inner.gcont)

$\Rightarrow k_{\text {_abstraction }}(\mathrm{fn}$ w $\Rightarrow$ pushcc $\mathrm{k}(\mathrm{fn}() \Rightarrow \mathrm{w}))$ )

end

$(*$ rule popcc, $*$ )

$(*$ side condition $(k s \neq$ nil $) *)$

$\left(* i d_{L}^{p o p} *\right)$

$(*$ rule pushcc, $*)$

(* rule reset $*$ )

$\left(*\right.$ rule $\left.\operatorname{shift}_{\mathrm{I}}^{*}\right)$

(* side condition $(k s \neq$ nil $) *)$

Fig. 2. A native implementation of the CPS hierarchy in Standard ML of New Jersey 


\section{Application: layering monadic effects}

As a significant application of composable continuations, Filinski's work on adding user-defined monadic effects to ML-like languages by monadic reflection shows that composing continuations is a universal effect, which can be used to simulate all effects expressible using a monad I2 I.3. The original work only allowed one monadic effect, but recently, Filinski has extended the technique to allow layering effects by relating a heterogeneous tower of monads to a tower of continuation monads, and then implementing them using a collection of cells to hold the meta-continuations 15 .

Independently, we directly adapted Filinski's original one-level implementation with minimal changes to parameterize the functor that generates a monad representation by the monad representation layered beneath it, which also gives an inductive implementation of a monadic hierarchy. We essentially put in each structure of a monad representation the corresponding level in the control hierarchy; the functor that generates an outer monad representation is passed the control level of the monad representation at the inner layer, and applies functor sr_outer to construct its own control level.

The benefits of this representation of layered monads is the same as in Filinski's work I5: it is a direct implementation, i.e., no level of interpretation and no level of translation hinder it 2636 .

More detail and several illustrative examples are available in the extended version of this article 7 .

\section{Related Work}

\subsection{Felleisen's seminal work}

As already mentioned in Section 12 the notion of control delimiters in direct

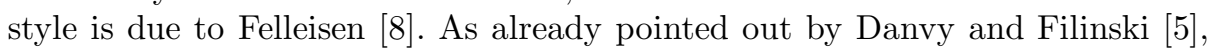
control delimiters are significant because they fit in each level of the CPS hierarchy very naturally: they correspond to resetting the current continuation to the identity function; and indeed the control delimiter reset is equivalent to Felleisen's. As for abstracting control, programming practice suggested the control operator shift which is equivalent to one of the variants of Felleisen's $\mathcal{F}$-operator.

Felleisen's work relies on a notion of control stack, and has inspired a number of similar control operators. Danvy and Filinski's work relies on CPS, and has inspired a number of applications, for two compound reasons we believe:

Expressiveness: Programming intuitions run strong in the world of control stacks. But lacking guidelines, how does one know, e.g., whether one has landed on [the continuation equivalent of] Algol 60's control stack or on Lisp's control stacki.e., on the control equivalent of lexical scope or on dynamic scope (whichever may be best)? And how does one use the result?

Conversely, the world of CPS is a structured one, which offers guidelines and holds much untapped expressive power. For example I2 I3, Filinski has 
shown that the expressive power of the CPS hierarchy is equivalent to the one of computational monads. In fact, our new examples could equally well be expressed using a tower of monads.

More specifically, operational descriptions of control hierarchies offer the possibilities to shadow control delimiters, to capture them or not when abstracting control, to restore them or not when reinstating abstracted control, and to dynamically search through them at run time. CPS shields us against the most extravagant of these mind-boggling possibilities, since by definition, programs with shift and reset denote CPS programs. These CPS programs may have many layers of continuations, but they are (1) purely functional and (2) statically typed.

Efficient implementation: A stack-based implementation of control tends to exert a cost which is linear in the use of each captured continuation. Besides, and this is a well-known thesis in the continuation community 3 , it faces a real problem of duplicated continuations.

Therefore alternative implementations have been sought. For example, Filinski already showed that shift $_{1}$ and reset $_{1}$ can be implemented concisely in terms of callcc, which itself can be implemented efficiently 312,0 . Through an alternative (but equivalent) formalism, our work essentially generalizes this concise implementation to the whole CPS hierarchy, with no new cost and an equivalent use.

\subsection{Filinski's work}

As a significant application of the CPS hierarchy, Filinski's work on adding userdefined monadic effects to ML-like languages by monadic reflection shows that composing continuations is a universal effect, which can be used to simulate all effects expressible using a monad 121315.

\subsection{Gunter, Rémy, and Riecke's work}

Gunter, Rémy, and Riecke present a new set of control operators generalizing exceptions and continuations, and its associated operational semantics and type system $1 \mathrm{~h}$. The strength of these operators lies in their static type systemin comparison, and even though we do not doubt that there is one for the CPS hierarchy (cf. Murthy's work 28 ), we do not present one here explicitly; instead, we rely on ML's type system in our implementation.

Independently of their type system, Gunter, Rémy, and Riecke's operators are not cast in stone. In their own words, "We do not feel, though, that there is a clear answer to the question of which operational rule is right; suffice it to say that we have picked one, and that the other rules lead to strong type soundness as well." Similarly, we do not contend that shift and reset are the ultimate control operators-Filinski's operators kreflect and kreify, for example, could well be preferred 1213 . But we do believe that the key to their simplicity and expressiveness is the CPS hierarchy.

Gunter, Rémy, and Riecke's operators are also implemented with callcc. 


\subsection{Operational semantics}

Operational semantics, especially small-step reduction semantics, is often used to specify control operators formally. Several researchers have investigated the type soundness of languages with control operators via syntactic approaches based on operational semantics, such as Wright and Felleisen, and Harper, Duba, and MacQueen for first-class continuations 17 39, Gunter, Rémy, and Riecke for generalizing exceptions and continuations 16, and Murthy for the CPS hierarchy 28 . Here, we use operational semantics to derive our implementation and to prove its correctness. Also, matching the CPS hierarchy, we present a family of continuation semantics instead of one monolithic semantics. This family can be natively programmed in ML without resorting to an informal notion of control stack.

\subsection{Continuations}

After 25 years of existence 32 , continuations still remain a challenging topic, to the point that ad-hoc frameworks are routinely preferred. For example, we find it significant that alternative and independent solutions were sought to compile goal-directed evaluation 30 and to abstract delimited control 816 , even though two levels of continuations provide a simple, natural, and directly implementable solution to both problems. This indicates that continuations require more basic research. We have tried to contribute to this research by characterizing a specific notion of operational continuation semantics and by formalizing its connection to the traditional CPS transformation.

\section{Conclusion}

The CPS transformation is ubiquitous in many areas of computer science, including logic, constructive mathematics, programming languages, and programming. Iterating it yields a concise and expressive framework for delimiting and abstracting control - the CPS hierarchy — which appears substantial and fruitful but has been explored very little so far. In this article, we have contributed to exploring it by (1) characterizing an operational analogue of continuation semantics; (2) developing an analogue of the CPS transformation for such an operational continuation semantics; (3) making it account for the family of control operators shift and reset; (4) providing a native implementation of the CPS hierarchy in the statically typed language Standard ML; and (5) illustrating the implementation both with classical and with new applications, and in particular with a direct implementation of layered monads.

\section{Acknowledgments}

Part of this work was carried out while the second author was visiting the BRICS $\mathrm{PhD}$ school at the University of Aarhus in the fall of 1997.

Thanks are due to Andrzej Filinski, Julia Lawall, and the anonymous referees for comments on an earlier version of this article. 


\section{References}

1. Andrew W. Appel and David B. MacQueen. Standard ML of New Jersey. In Jan Maluszyński and Martin Wirsing, editors, Third International Symposium on Programming Language Implementation and Logic Programming, number 528 in Lecture Notes in Computer Science, pages 1-13, Passau, Germany, August 1991. Springer-Verlag.

2. Robert (Corky) Cartwright, editor. Proceedings of the 1988 ACM Conference on Lisp and Functional Programming, Snowbird, Utah, July 1988. ACM Press.

3. William Clinger, Anne H. Hartheimer, and Eric M. Ost. Implementation strategies for first-class continuations. Higher-Order and Symbolic Computation, 12(1), 1999.

4. Olivier Danvy. Type-directed partial evaluation. In Guy L. Steele Jr., editor, Proceedings of the Twenty-Third Annual ACM Symposium on Principles of Programming Languages, pages 242-257, St. Petersburg Beach, Florida, January 1996. ACM Press.

5. Olivier Danvy and Andrzej Filinski. Abstracting control. In Wand 38, pages 151-160.

6. Olivier Danvy and Andrzej Filinski. Representing control, a study of the CPS transformation. Mathematical Structures in Computer Science, 2(4):361-391, December 1992.

7. Olivier Danvy and Zhe Yang. An operational investigation of the CPS hierarchy (extended version). Technical Report BRICS RS-98-35, Department of Computer Science, University of Aarhus, Aarhus, Denmark, December 1998.

8. Matthias Felleisen. The theory and practice of first-class prompts. In Ferrante and Mager II, pages 180-190.

9. Matthias Felleisen, Daniel P. Friedman, Bruce Duba, and John Merrill. Beyond continuations. Technical Report 216, Computer Science Department, Indiana University, Bloomington, Indiana, February 1987.

10. Matthias Felleisen, Mitchell Wand, Daniel P. Friedman, and Bruce F. Duba. Abstract continuations: A mathematical semantics for handling full functional jumps. In Cartwright 2, pages 52-62.

11. Jeanne Ferrante and Peter Mager, editors. Proceedings of the Fifteenth Annual ACM Symposium on Principles of Programming Languages, San Diego, California, January 1988. ACM Press.

12. Andrzej Filinski. Representing monads. In Hans-J. Boehm, editor, Proceedings of the Twenty-First Annual ACM Symposium on Principles of Programming Languages, pages 446-457, Portland, Oregon, January 1994. ACM Press.

13. Andrzej Filinski. Controlling Effects. PhD thesis, School of Computer Science, Carnegie Mellon University, Pittsburgh, Pennsylvania, May 1996. Technical Report CMU-CS-96-119.

14. Andrzej Filinski. From normalization-by-evaluation to type-directed partial evaluation. In Olivier Danvy and Peter Dybjer, editors, Preliminary Proceedings of the 1998 APPSEM Workshop on Normalization by Evaluation, NBE '98, (Chalmers, Sweden, May 8-9, 1998), number NS-98-1 in BRICS Note Series, Department of Computer Science, University of Aarhus, May 1998.

15. Andrzej Filinski. Representing layered monads. In Alex Aiken, editor, Proceedings of the Twenty-Sixth Annual ACM Symposium on Principles of Programming Languages, San Antonio, Texas, January 1999. ACM Press. To appear.

16. Carl Gunter, Didier Rémy, and Jon G. Riecke. A generalization of exceptions and control in ML-like languages. In Simon Peyton Jones, editor, Proceedings of the 
Seventh ACM Conference on Functional Programming and Computer Architecture, pages 12-23, La Jolla, California, June 1995. ACM Press.

17. Robert Harper, Bruce F. Duba, and David MacQueen. Typing first-class continuations in ML. Journal of Functional Programming, 3(4):465-484, October 1993.

18. Robert Hieb and R. Kent Dybvig. Continuations and concurrency. In Proceedings of the Second ACM SIGPLAN Symposium on Principles 85 Practice of Parallel Programming, SIGPLAN Notices, Vol. 25, No. 3, pages 128-136, Seattle, Washington, March 1990. ACM Press.

19. Robert Hieb, R. Kent Dybvig, and Claude W. Anderson, III. Subcontinuations. Lisp and Symbolic Computation, 5(4):295-326, December 1993.

20. Robert Hieb, R. Kent Dybvig, and Carl Bruggeman. Representing control in the presence of first-class continuations. In Bernard Lang, editor, Proceedings of the ACM SIGPLAN'90 Conference on Programming Languages Design and Implementation, SIGPLAN Notices, Vol. 25, No 6, pages 66-77, White Plains, New York, June 1990. ACM Press.

21. Gregory F. Johnson. GL - a denotational testbed with continuations and partial continuations as first-class objects. In Mark Scott Johnson, editor, Proceedings of the ACM SIGPLAN'87 Symposium on Interpreters and Interpretive Techniques, SIGPLAN Notices, Vol. 22, No 7, pages 154-176, Saint-Paul, Minnesota, June 1987. ACM Press.

22. Gregory F. Johnson and Dominic Duggan. Stores and partial continuations as first-class objects in a language and its environment. In Ferrante and Mager II , pages $158-168$.

23. Richard Kelsey, William Clinger, and Jonathan Rees, editors. Revised ${ }^{5}$ report on the algorithmic language Scheme. Higher-Order and Symbolic Computation, 11(1):7-105, 1998. Also appears in ACM SIGPLAN Notices 33(9), September 1998.

24. Julia L. Lawall. Continuation Introduction and Elimination in Higher-Order Programming Languages. PhD thesis, Computer Science Department, Indiana University, Bloomington, Indiana, July 1994.

25. Julia L. Lawall and Olivier Danvy. Continuation-based partial evaluation. In Carolyn L. Talcott, editor, Proceedings of the 1994 ACM Conference on Lisp and Functional Programming, LISP Pointers, Vol. VII, No. 3, Orlando, Florida, June 1994. ACM Press.

26. Sheng Liang, Paul Hudak, and Mark Jones. Monad transformers and modular interpreters. In Peter Lee, editor, Proceedings of the Twenty-Second Annual ACM Symposium on Principles of Programming Languages, pages 333-343, San Francisco, California, January 1995. ACM Press.

27. Luc Moreau and Christian Queinnec. Partial continuations as the difference of continuations, a duumvirate of control operators. In Manuel Hermenegildo and Jaan Penjam, editors, Sixth International Symposium on Programming Language Implementation and Logic Programming, number 844 in Lecture Notes in Computer Science, pages 182-197, Madrid, Spain, September 1994. Springer-Verlag.

28. Chethan R. Murthy. Control operators, hierarchies, and pseudo-classical type systems: A-translation at work. In Olivier Danvy and Carolyn L. Talcott, editors, Proceedings of the ACM SIGPLAN Workshop on Continuations, Technical report STAN-CS-92-1426, Stanford University, pages 49-72, San Francisco, California, June 1992.

29. Gordon D. Plotkin. Call-by-name, call-by-value and the $\lambda$-calculus. Theoretical Computer Science, 1:125-159, 1975. 
30. Todd A. Proebsting. Simple translation of goal-directed evaluation. In Ron K. Cytron, editor, Proceedings of the ACM SIGPLAN'97 Conference on Programming Languages Design and Implementation, SIGPLAN Notices, Vol. 32, No 5, pages 1-6, Las Vegas, Nevada, June 1997. ACM Press.

31. Christian Queinnec and Bernard Serpette. A dynamic extent control operator for partial continuations. In Robert (Corky) Cartwright, editor, Proceedings of the Eighteenth Annual ACM Symposium on Principles of Programming Languages, pages 174-184, Orlando, Florida, January 1991. ACM Press.

32. John C. Reynolds. The discoveries of continuations. Lisp and Symbolic Computation, 6(3/4):233-247, December 1993.

33. Dorai Sitaram. Handling control. In David W. Wall, editor, Proceedings of the ACM SIGPLAN'93 Conference on Programming Languages Design and Implementation, SIGPLAN Notices, Vol. 28, No 6, pages 147-155, Albuquerque, New Mexico, June 1993. ACM Press.

34. Dorai Sitaram and Matthias Felleisen. Control delimiters and their hierarchies. Lisp and Symbolic Computation, 3(1):67-99, January 1990.

35. Dorai Sitaram and Matthias Felleisen. Reasoning with continuations II: Full abstraction for models of control. In Wand 38, pages 161-175.

36. Philip Wadler. The essence of functional programming (tutorial). In Andrew W. Appel, editor, Proceedings of the Nineteenth Annual ACM Symposium on Principles of Programming Languages, pages 1-14, Albuquerque, New Mexico, January 1992. ACM Press.

37. Philip Wadler. Monads and composable continuations. LISP and Symbolic Computation, 7(1):39-55, January 1994.

38. Mitchell Wand, editor. Proceedings of the 1990 ACM Conference on Lisp and Functional Programming, Nice, France, June 1990. ACM Press.

39. Andrew K. Wright and Matthias Felleisen. A syntactic approach to type soundness. Information and Computation, 115:38-94, 1994. 